\title{
Productive Performance, Egg Characteristics and Hatching Traits of Three Chicken Genotypes under Free-Range, Semi-Intensive, and Intensive Housing Systems
}

Original Article

EAuthor(s)

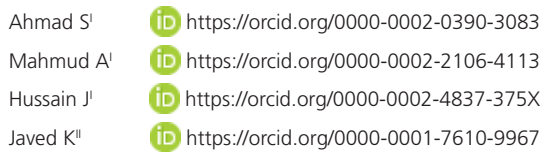

Department of Poultry Production, Faculty of Animal Production and Technology, University of Veterinary and Animal Sciences, Lahore, 54000, Pakistan.

Department of Livestock Production, Faculty of Animal Production and Technology, University of Veterinary and Animal Sciences, Lahore, 54000, Pakistan.

\section{-Mail Address}

Corresponding author e-mail address Sohail Ahmad

Department of Poultry Production, Faculty of Animal Production and Technology,

University of Veterinary and Animal

Sciences, Lahore, Punjab, Pakistan.

Phone: +92-313-4288381

Email: sohail.ahmad@uvas.edu.pk

\section{- Keywords}

Crossbred chickens, egg quality, hatching traits, housing system, live performance.

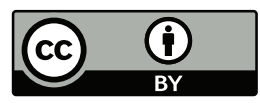

Submitted: 04/November/2018 Approved: 19/February/2019

\section{ABSTRACT}

The present study aimed at evaluating the effect of housing system on the live performance, egg quality, and hatching traits of three dual-purpose chicken genotypes. In total, 180 birds, comprising 48 pullets and 12 cockerels from each of three genotypes, were evaluated during the production phase (27-46 weeks). For this, 144 pullets and 36 cockerels were randomly picked from 18 treatment block groups were shifted to breeding coops, allotting 4 pullets to one cockerel. A completely randomized complete block design (RCBD) was employed. Three genotypes, purebred Naked Neck (NN) and two crosses Rhode Island Red $\times$ Naked Neck (RIR $\times$ NN $=$ RNN) and Black Australorp $\times$ Naked Neck (BAL $\times \mathrm{NN}=\mathrm{BNN})$, were compared. Intensive system $(p<0.0001)$ and BNN hens $(p<0.0001)$ were heavier on week 26 and 46 . Higher egg production $(p<0.0001)$ was obtained in the intensive system and in BNN hens $(p<0.0001)$. Hens maintained in the intensive systems produced heavier eggs and higher egg mass $(p<0.0001)$, and RNN and BNN hens laid heavier eggs $(p<0.0001)$ while higher egg mass $(p<0.0001)$ was found in BNN hens. Higher egg shape index (initial, $p=0.0002$ ), egg surface area (initial, $p<0.0001$; final, $p<0.0001$ ), egg volume (initial, $p<0.0001$; final, $p<0.0001$ ) and Haugh unit score (initial, $p=0.0002$; final, $p=<0.0001$ ) were obtained in RNN and BNN hens. At the end of the experiment (46 weeks), higher yolk index $(p=0.0004)$ was found in RNN and BNN eggs, and thicker eggshells $(p<0.0001)$ in RNN eggs. Higher egg hatchability was obtained in the free-range system $(p<0.0001)$ and in the RNN genotype $(p<0.0001)$. The highest fertility rates were detected in the free-range system $(p<0.0001)$, and in the RNN and BNN genotypes $(p<0.0001)$. The lowest infertile egg rates were observed in the free-range system $(p<0.0001)$ and in RNN and BNN genotypes $(p<0.0001)$. The lowest dead-in-shell rate was calculated for the free-range system $(p=0.0456)$. In conclusion, free-range and semiintensive system largely influence productive performance, egg quality and hatching traits. Regarding genotypes, RNN and BNN crossbred hens perform better than NN purebreds.

\section{INTRODUCTION}

In Pakistan, indigenous chickens are reared in rural and peri-urban areas for egg and meat production, which are sources of high-quality protein and also contribute for the nation's GDP (Economic Survey, 201718). Indigenous chicken breeds include Aseel, Desi (non-descript) and Naked-Neck; however, some exotic breeds, such as Black Australorp, Fayoumi, Rhode Island Red and their crosses are also reared by the rural farmers (Sadef et al., 2015). Indigenous chicken breeds have become increasingly popular around the globe due to their better adaptability to local environmental conditions and good immune profile (lqbal et al., 2012). 
Egg quality is a major consideration in the egg industry and it is influenced by consumer demands. Furthermore, internal egg content also affects hatching results, particularly chick yield (Rehman et al., 2017). The development of embryonic tissues and efficient hatching require good albumen and yolk quality and are influenced by egg morphometrics. Eggshell thickness is also an important parameter in this regard, as eggshells must be free from any deformities for ideal gas exchange and pipping process, and from hair-like cracks to avoid unnecessary moisture loss. That is the reason why most breeding companies focus on egg-quality traits (Bain, 2005; Sekeroglu \& Altuntas, 2009).

Fertility and hatchability are the major constraints that affect the profitability of the hatchery industry and are influenced by genetics, physiology, and extrinsic factors. Peter et al. (2008) reported fertility variation among different chicken genotypes and found comparable semen quality and quantity in local Nigerian and exotic chickens. In a study of three exotic and one indigenous chickens of Ethiopia, the highest hatchability (79\%) was recorded in indigenous chickens (Lemlem \& Tesfay, 2010). Similarly, in dualpurpose chicken genotypes, the highest fertility and hatchability were observed in both pure and crossbred Nigerian chicken genotypes and were attributed to gene segregation (Adeleke et al., 2012).

Housing systems have a substantial effect on live performance and egg quality traits; however, genotypes and feeding regimes are also considered as major factors influencing egg geometry and hatching traits (Chen et al., 2013). Over the last few years, people have become more concerned about the quality and welfare of poultry. In this regard, international regulations have been developed to minimize the use of conventional cage systems and to promote poultry welfare.

Since the ban on conventional cages in 2012 by European Union, producers have been highly motivated to find alternative housing systems, such as enriched cages and free-range and semi-intensive systems (Leinonen et al., 2014). Birds in free-range systems are allowed to graze on seasonal legume and grass pastures, which, in addition, provide earthworms to the birds. Free-range systems not only fulfill the welfare needs of the birds, but the availability of nutritious plants and worms also reduces total production cost (Lay et al., 2011).

Indigenous chickens of Pakistan are generally termed as scavengers; however, their performance in alternative production systems are still unclear. Therefore, present study aimed at evaluating the live performance, egg characteristics and hatching traits of three chicken genotypes (Rhode Island Red $\times$ Naked Neck, Black Australorp $\times$ Naked Neck, and Naked Neck $\times$ Naked Neck) reared under free-range, semi-intensive and intensive housing systems.

\section{MATERIALS AND METHODS}

This study was conducted at Department of Poultry Production, UVAS, A-Block, Ravi Campus, Pattoki, Pakistan. Pattoki is located at $31^{\circ} 1^{\prime} \mathrm{ON}$ and $73^{\circ} 50^{\prime} 60 \mathrm{E}$ with an altitude of $186 \mathrm{~m}$ (610 ft). This city experiences normally hot and humid tropical climate with maximum temperature ranging from $13^{\circ} \mathrm{C}$ in winter and $43^{\circ} \mathrm{C}$ in summer.

\section{Ethics}

Bird care and use were in accordance with the laws and regulations of Pakistan and was approved by Committee of Ethical Handling of Experimental Birds (No. DR/124), University of Veterinary and Animal Sciences (UVAS), Pakistan.

\section{Experimental birds}

One hundred and sixty one-day-old of each genotype: Rhode Island Red $\times$ Naked Neck (RNN), Black Australorp $\times$ Naked Neck (BNN) and Naked Neck $\times$ Naked Neck (NN), totaling 480 chicks, hatched at Avian Research and Training Centre, UVAS, Lahore, Pakistan, were transported to the Indigenous Chicken Genetic Resource Centre (ICGRC), A-Block, UVAS, Ravi Campus, Pattoki.

Chicks were housed in floor pens in a wellventilated open-sided shed and submitted to standard management conditions until 6 weeks of age. Birds were fed a commercial broiler breeder diet (16\% crude protein (CP), $2900 \mathrm{kcal}$ metabolizable energy (ME)/kg). During the brooding period, birds were vaccinated against Newcastle Disease (ND) and Infectious Bronchitis (IB), according to the local schedule.

From 7 to 16 weeks, birds were fed with grower diet (20.02\% crude protein and $3020 \mathrm{kcal}$ metabolizable energy $(\mathrm{ME}) / \mathrm{kg})$. Morphometric traits were evaluated on weekly basis. At the end of 16 weeks, three birds from each treatment group were randomly collected and slaughtered according to Halal ritual to record carcass traits (Ahmad et al., 2019a).

At 16 weeks of age, out of total of 260 birds (156 pullets and 104 cockerels) remaining from the growing 
phase, 156 pullets and 39 cockerels were randomly selected and evaluated during the development phase (16 to 27 weeks of age). Birds were fed with a commercial diet (15\% CP, $2750 \mathrm{kcal} \mathrm{ME} / \mathrm{kg})$ and their morphometric traits, blood biochemical profile and antibody responses were evaluated (Ahmad et al., 2019b).

At 27 weeks of age, 180 birds (48 pullets and 12 cockerels of each of the three crosses) were evaluated during the rearing phase. For the egg-production phase, 144 pullets and 36 cockerels were randomly selected from the 18 treatment block groups ( 3 genotypes $\times 3$ housing systems $\times 2$ sexes) and transferred to laying cages or breeding pens, allotting four pullets per cockerel. Pen-mating system was applied to obtain fertile eggs.

\section{Free-range, Semi-intensive and Intensive Systems}

All experimental birds were individually tagged and maintained in an open-sided shed $(\mathrm{L} \times \mathrm{W} \times \mathrm{H})$ oriented east to west. A patch of fertile land measuring $L \times W$ (stocking density $=0.23 / \mathrm{m}^{2}$ ) located in front of the shed was used as range area. The free-range area was enriched with grass and legume species [mung (Vigna radiata L.), black-eyed pea (Vigna unguiculata L.), French pea (Phaseolus vulgaris L.), and alfalfa (Medicago sativa L.)]. The range area was divided into two rows using fishing nets (one for free-range and other for semi-intensive). Fresh ad libitum water was ensured through manual drinkers. For the protection of the birds, a $2.44 \mathrm{~m}$-high wire mesh enclosure was placed surrounding the range area. In the free-range and semi-intensive systems, birds were given access to vegetation and drinking water from 06:00 to 18:00 $\mathrm{h}$, and $06: 00$ to $12: 00 \mathrm{~h}$, respectively (Figure 1).

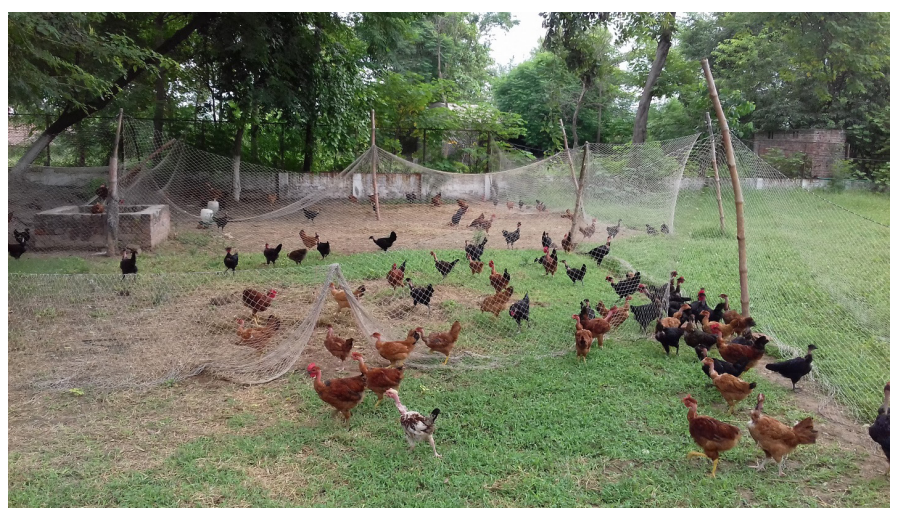

Figure 1 - Birds in range area

In the intensive system, the laying birds were kept at well-ventilated poultry shed equipped with a three-tier battery cage system with a steep wire floor to facilitate egg collection (FACCO, Poultry Equipment-C3). Under the floor of the cages, dropping belts were placed to collect the fecal material. Floor space of $0.19 \mathrm{~m}^{2}$ per bird was provided.

\section{Experimental diets}

The laying hens under the free-range feeding system were offered $100 \mathrm{~g}$ of a mixture of seasonal legumes, beans, herbs, and range shrubs twice a day, and were supplemented with a laying breeder feed at $25 \%$ of the standard feed allowance (Table 1). Birds in the semi-intensive housing system were also offered the same $100 \mathrm{~g}$ of the plant mixture, but once daily and the remaining nutritional requirements were supplied by offering $50 \%$ of the standard feed allowance. The birds in the intensive housing system were offered a laying breeder diet as per recommendation of the NRC (1994) and Leeson \& Summers (2005) (Table 1).

Table 1 - Ingredient and nutrient composition of experimental ration.

\begin{tabular}{lcc}
\hline Feed Ingredient (\%) & Female formulation (\%) & Male formulation (\%) \\
\hline Corn & 42.61 & 39.4 \\
SBM & 15.62 & 10.45 \\
Corn Gluten (60\%) & 1 & -- \\
Rice Tips & 19 & 31 \\
Wheat Bran & 13 & 15.8 \\
DCP & 1.2 & 0.70 \\
$\mathrm{CaCO}_{3}$ & 7.42 & 2.65 \\
DL-Methionine & 0.15 & -- \\
Nutrient & & \\
Crude Protein & 15.04 & 13.13 \\
ME (Kcal/kg) & 2682 & 2848 \\
Calcium & 2.81 & 1.09 \\
Phosphorus & 0.34 & 0.22 \\
Lysine & 0.86 & 0.74 \\
Methionine & 0.45 & 0.39 \\
\hline
\end{tabular}

(Leeson \& Summers, 2005).

\section{Parameters evaluated}

Eggs were collected daily to calculate hen day production percentage (Shafik et al., 2013), egg weight (g) and egg mass (g). Eggs were stored for 7 days at $13-15^{\circ} \mathrm{C}$ and $70-80 \%$ relative humidity, Eggs were set in the of the Avian Research and Training Centre, UVAS, Lahore, under standard conditions (Victoria Inc.) in order to evaluate hatching results (hatchability, fertility, early embryonic mortality and late embryonic mortality) as adopted by Adeleke et al. (2012).

A total of 45 eggs, comprising five eggs per treatment group, were evaluated for morphometry and quality traits at the start and at the end of experiment, according to the methods adopted by Gikunju et al. (2018). 


\section{Statistical Analysis}

The obtained performance, egg characteristics, and hatching trait data were analyzed by two-way analysis of variance, assuming genotypes and housing systems as adjusted effects, applying the General Linear Model procedures of SAS software. Treatment means were separated by Tukey's HSD test (Tukey, 1953) considering significance level of $P \leq 0.05$. The following mathematical model was used:

$Y_{i j k}=\mu+\beta_{i}+\tau_{j}+(\beta \times \tau)_{i j}+\epsilon_{i j k}$

Where,

$Y_{\mathrm{j} j \mathrm{k}}=$ Observation of dependent variable recorded on $\mathrm{j}^{\text {th }}$ housing system in $\mathrm{i}^{\text {th }}$ block

$\mu=$ Population mean

$\beta_{i}=$ Effect of $i^{\text {th }}$ block (genotype; $i=1,2,3$ )

$\tau_{j}=$ Effect of $j^{\text {th }}$ housing system $(j=1,2,3)$

$(\beta \times \tau)_{\mathrm{ij}}=$ Interaction between genotype and housing system

$\epsilon_{i j \mathrm{k}}=$ Residual error of $\mathrm{k}^{\text {th }}$ observation on $\mathrm{j}^{\text {th }}$ treatment in $i^{\text {th }}$ block NID $\sim 0, \sigma^{2}$

\section{RESULTS}

\section{Productive Performance}

Productive performance differed among housing systems, genotypes and their interaction (Tables 2, 3).
Hens maintained in the intensive system were heavier on weeks $26(p<0.0001)$ and $46(p<0.0001)$ than those in the semi-intensive and free-range systems. Similarly, BNN hens were heavier on week $26(p<0.0001)$ and $46(p=0.0025)$ compared with RNN and NN hens. In weeks $26(p<0.0001)$ and $46(p<0.0001)$, BNN hens in the semi-intensive system were heavier than those of the other genotypes.

Hen day production \% was higher $(p<0.0001)$ in the intensive system than in the free-range and semi-intensive systems. Relative to genotypes, egg production \% was higher $(p<0.0001)$ in BNN hens followed by RNN and NN. The interaction between housing systems and genotype $(p<0.0001)$ showed higher egg production \% in RNN and BNN hens kept in the semi-intensive system and BNN chickens with free-range system.

Hens kept in the intensive system produced heavier eggs $(p<0.0001)$ followed by the semi-intensive and free-range systems. Among genotypes, RNN and BNN hens produced heavier eggs $(p<0.0001)$ than $N N$ hens. In the interaction between housing systems and genotypes, higher $(p<0.0001)$ egg weight was found in RNN and BNN hens kept in the intensive and semiintensive systems.

Higher egg mass $(p<0.0001)$ was obtained in the intensive system, followed by the semi-intensive and

Table 2 - Effect of genotype and housing system on productive performance (26-46 weeks). ${ }^{1}$

\begin{tabular}{|c|c|c|c|c|c|c|c|c|}
\hline \multirow{2}{*}{ Trait } & \multicolumn{3}{|c|}{ Genotype } & \multirow{2}{*}{ P-value } & \multicolumn{3}{|c|}{ Housing System } & \multirow{2}{*}{$p$-value } \\
\hline & $\operatorname{RNN}(n=48)$ & BNN $(n=48)$ & $N N(n=48)$ & & $F R(n=48)$ & $\mathrm{SI}(\mathrm{n}=48)$ & $I(n=48)$ & \\
\hline BW 26wk & $1228.36^{b} \pm 30.52$ & $1366.66^{a} \pm 40.62$ & $1064.75^{`} \pm 30.63$ & $<0.0001$ & $1082.16^{\complement} \pm 16.93$ & $1215.59^{b} \pm 51.65$ & $1362.03^{\mathrm{a}} \pm 30.77$ & $<0.0001$ \\
\hline BW 46wk & $1484.45^{b} \pm 100.73$ & $1679.74^{a} \pm 99.35$ & $1391.25^{b} \pm 72.91$ & 0.0025 & $1171.86^{c} \pm 31.19$ & $1467.94^{b} \pm 34.74$ & $1915.64^{a} \pm 95.95$ & $<0.0001$ \\
\hline$P R$ & $60.21^{b} \pm 0.14$ & $60.71^{a} \pm 0.18$ & $54.13^{c} \pm 0.29$ & $<0.0001$ & $57.80^{b} \pm 0.59$ & $57.56^{b} \pm 0.43$ & $59.70^{a} \pm 0.35$ & $<0.0001$ \\
\hline EW & $53.16^{a} \pm 0.15$ & $53.13^{a} \pm 0.14$ & $46.68^{b} \pm 0.06$ & $<0.0001$ & $50.11^{c} \pm 0.36$ & $51.31^{b} \pm 0.49$ & $51.54^{a} \pm 0.49$ & $<0.0001$ \\
\hline CEM & $4.79^{b} \pm 0.02$ & $4.82^{\mathrm{a}} \pm 0.02$ & $3.80^{\circ} \pm 0.02$ & $<0.0001$ & $4.35^{c} \pm 0.07$ & $4.44^{b} \pm 0.07$ & $4.62^{\mathrm{a}} \pm 0.07$ & $<0.0001$ \\
\hline Liv & $99.98 \pm 0.01$ & $99.96 \pm 0.01$ & $99.97 \pm 0.01$ & 0.7898 & $99.98 \pm 0.01$ & $99.95 \pm 0.02$ & $99.98 \pm 0.01$ & 0.1141 \\
\hline
\end{tabular}

a-c Means in a row with no common superscript differ significantly at $p \leq 0.05$.

'Values are least square mean \pm standard error.

RNN = Rhode Island Red $\times$ Naked Neck; BNN = Black Australorp $\times$ Naked Neck; NN = Naked Neck; FR = Free Range; SI = Semi Intensive; I = Intensive; BW = Body Weight (g); wk $=$ week; PR = Production \%; EW = Egg Weight (g); CEM = Cumulative Egg Mass per bird (Kg); Liv= Livability \%.

Table 3 - Interaction effects (genotype $\times$ housing system) on productive performance (26-46 weeks) ${ }^{1}$.

\begin{tabular}{|c|c|c|c|c|c|c|c|c|c|c|}
\hline \multirow{2}{*}{ Trait } & \multicolumn{3}{|c|}{ RNN } & \multicolumn{3}{|c|}{ BNN } & \multicolumn{3}{|c|}{$\mathrm{NN}$} & \multirow{2}{*}{$p$-value } \\
\hline & $F R(n=16)$ & $S I(n=16)$ & $I(n=16)$ & $F R(n=16)$ & $\mathrm{SI}(\mathrm{n}=16)$ & $I(n=16)$ & $\mathrm{FR}(\mathrm{n}=16)$ & $\mathrm{SI}(\mathrm{n}=16)$ & $I(n=16)$ & \\
\hline BW 26wk & $1074.54^{e} \pm 15.55$ & $1251.15^{c} \pm 23.82$ & $1359.40^{\mathrm{b}} \pm 20.60$ & $1163.73^{d} \pm 14.35$ & $1430.93^{\mathrm{ab}} \pm 53.39$ & $1505.32^{a} \pm 29.37$ & $1008.22^{\mathrm{ef}} \pm 6.41$ & $964.68^{f} \pm 40.90$ & $1221.36^{\mathrm{cd}} \pm 17.49$ & $<0.0001$ \\
\hline BW 46wk & $1157.92^{\mathrm{ef}} \pm 31.66$ & $1369.80 d^{e} \pm 49.82$ & $1925.63^{\mathrm{ab}} \pm 192.66$ & $1318.34^{\text {def }} \pm 32.14$ & $1606.50^{\text {cd }} \pm 36.42$ & $2114.37^{a} \pm 182.59$ & $1039.31^{\dagger} \pm 6.60$ & $1427.51^{\text {cde }} \pm 49.07$ & $1706.92^{\mathrm{bc}} \pm 82.50$ & $<0.0001$ \\
\hline PR & $60.09^{b} \pm 0.15$ & $59.43^{c} \pm 0.15$ & $61.11^{\mathrm{a}} \pm 0.23$ & $61.07^{a} \pm 0.20$ & $59.67^{\mathrm{bc}} \pm 0.29$ & $61.40^{\mathrm{a}} \pm 0.26$ & $52.24^{f} \pm 0.20$ & $53.57 \mathrm{e} \pm 0.14$ & $56.60^{d} \pm 0.26$ & $<0.0001$ \\
\hline EW & $51.81^{b} \pm 0.12$ & $53.74^{a} \pm 0.08$ & $53.92^{\mathrm{a}} \pm 0.10$ & $51.87^{b} \pm 0.11$ & $53.66^{\mathrm{a}} \pm 0.06$ & $53.85^{\mathrm{a}} \pm 0.09$ & $46.65^{\mathrm{cd}} \pm 0.12$ & $46.53^{d} \pm 0.06$ & $46.85^{\complement} \pm 0.10$ & $<0.0001$ \\
\hline CEM & $4.67^{c} \pm 0.02$ & $4.77^{\mathrm{b}} \pm 0.02$ & $4.92^{\mathrm{a}} \pm 0.02$ & $4.74^{b} \pm 0.02$ & $4.78^{b} \pm 0.02$ & $4.94^{\mathrm{a}} \pm 0.02$ & $3.66^{f} \pm 0.02$ & $3.76^{\mathrm{e}} \pm 0.01$ & $3.99^{d} \pm 0.02$ & 0.0036 \\
\hline Liv & $99.98 \pm 0.01$ & $99.96 \pm 0.03$ & $99.90 \pm 0.01$ & $99.97 \pm 0.02$ & $99.94 \pm 0.04$ & $99.98 \pm 0.01$ & $99.99 \pm 0.01$ & $99.95 \pm 0.02$ & $99.98 \pm 0.02$ & 0.7423 \\
\hline
\end{tabular}

a-f Means in a row with no common superscript differ significantly at $p \leq 0.05$.

1 Values are least square mean \pm standard error.

RNN = Rhode Island Red $\times$ Naked Neck; BNN = Black Australorp $\times$ Naked Neck; NN = Naked Neck; FR = Free Range; SI = Semi Intensive; I = Intensive; BW = Body Weight (g); wk = week; PR = Production \%; EW = Egg Weight (g); CEM = Cumulative Egg Mass per bird (Kg); Liv= Livability \%. 
free-range systems. As for the effect of genotypes, higher $(p<0.0001)$ egg mass was determined in BNN hens, followed by RNN and NN hens. The interaction between housing system and genotype $(p=0.0036)$ resulted in higher egg mass in RNN and BNN hens reared in the semi-intensive and intensive systems.

Mean livability did not differ among housing systems ( $p=0.1141)$ or genotypes $(p=0.7898)$ and no significant interaction ( $p=0.7423$ ) between factors was detected.

\section{Egg characteristics}

Egg morphometry and quality traits of genotypes and their interaction with housing system showed several differences (Table 4, 5, 6, 7).

Initially, egg shape index was higher $(p=0.0002)$ in RNN and BNN hens than in NN hens. The interaction ( $p=0.0053$ ) between housing systems and genotypes showed that RNN chickens in the semi-intensive system had the highest egg shape index. At the end of the

Table 4 - Effect of genotype and housing system on egg characteristics at 26 weeks. ${ }^{1}$

\begin{tabular}{|c|c|c|c|c|c|c|c|c|}
\hline \multirow{2}{*}{ Trait } & \multicolumn{3}{|c|}{ Genotype } & \multirow{2}{*}{$p$-value } & \multicolumn{3}{|c|}{ Housing System } & \multirow{2}{*}{$p$-value } \\
\hline & RNN $(n=15)$ & BNN $(n=15)$ & $N N(n=15)$ & & $F R(n=15)$ & $\mathrm{SI}(\mathrm{n}=15)$ & $I(n=15)$ & \\
\hline SI & $74.24^{a} \pm 0.42$ & $73.98^{a} \pm 0.38$ & $71.91^{b} \pm 0.34$ & 0.0002 & $73.48 \pm 0.39$ & $73.33 \pm 0.60$ & $73.32 \pm 0.39$ & 0.9503 \\
\hline SA & $58.24^{a} \pm 0.42$ & $58.13^{a} \pm 0.36$ & $55.78^{b} \pm 0.35$ & $<0.0001$ & $57.55 \pm 0.42$ & $57.31 \pm 0.45$ & $57.28 \pm 0.57$ & 0.8667 \\
\hline EV & $40.92^{a} \pm 0.44$ & $40.81^{a} \pm 0.38$ & $38.37^{b} \pm 0.36$ & $<0.0001$ & $40.21 \pm 0.43$ & $39.95 \pm 0.46$ & $39.93 \pm 0.60$ & 0.8695 \\
\hline EW & $44.82^{a} \pm 0.48$ & $44.70^{a} \pm 0.41$ & $42.02^{b} \pm 0.40$ & $<0.0001$ & $44.04 \pm 0.47$ & $43.76 \pm 0.51$ & $43.74 \pm 0.65$ & 0.8702 \\
\hline $\mathrm{HU}$ & $78.84^{a} \pm 0.75$ & $77.23^{a} \pm 0.66$ & $74.56^{b} \pm 0.39$ & 0.0002 & $76.53 \pm 0.78$ & $76.93 \pm 0.82$ & $77.17 \pm 0.72$ & 0.7844 \\
\hline YI & $49.65 \pm 1.14$ & $50.00 \pm 0.76$ & $47.94 \pm 0.23$ & 0.1883 & $49.93 \pm 0.98$ & $49.42 \pm 0.82$ & $48.25 \pm 0.59$ & 0.3512 \\
\hline ST & $0.33^{\mathrm{ab}} \pm 0.01$ & $0.32^{b} \pm 0.01$ & $0.34^{\mathrm{a}} \pm 0.01$ & 0.0787 & $0.34 \pm 0.01$ & $0.33 \pm 0.01$ & $0.32 \pm 0.01$ & 0.0724 \\
\hline
\end{tabular}

${ }^{a-b}$ Means in a row with no common superscript differ significantly at $p \leq 0.05$.

'Values are least square mean \pm standard error.

RNN = Rhode Island Red $\times$ Naked Neck; BNN = Black Australorp $\times$ Naked Neck; NN = Naked Neck; FR = Free Range; SI = Semi Intensive; I = Intensive; SI = Shape Index; SA = Surface Area $\left(\mathrm{cm}^{2}\right) ; \mathrm{EV}=$ Egg Volume $\left(\mathrm{cm}^{3}\right)$; EW = Egg Weight (g); HU = Haugh Unit Score; YI = Yolk Index; ST = Shell Thickness (mm).

Table 5 - Interaction effects (genotype $\times$ housing system) on egg characteristics at 26 weeks ${ }^{1}$.

\begin{tabular}{|c|c|c|c|c|c|c|c|c|c|c|}
\hline \multirow{2}{*}{ Trait } & \multicolumn{3}{|c|}{ RNN } & \multicolumn{3}{|c|}{ BNN } & \multicolumn{3}{|c|}{ NN } & \multirow{2}{*}{$p$-value } \\
\hline & $\mathrm{FR}(\mathrm{n}=5)$ & $\mathrm{SI}(\mathrm{n}=5)$ & $I(n=5)$ & $\mathrm{FR}(\mathrm{n}=5)$ & $\mathrm{SI}(\mathrm{n}=5)$ & $I(n=5)$ & $\mathrm{FR}(\mathrm{n}=5)$ & $\mathrm{SI}(n=5)$ & $I(n=5)$ & \\
\hline SI & $73.93^{\mathrm{ab}} \pm 0.26$ & $75.17^{a} \pm 0.76$ & $73.63^{\mathrm{ab}} \pm 0.93$ & $74.33^{\mathrm{ab}} \pm 0.69$ & $73.52^{\mathrm{ab}} \pm 0.86$ & $74.09^{a b} \pm 0.44$ & $72.27^{b c} \pm 0.64$ & $71.31^{\complement} \pm 0.80$ & $72.24^{b c} \pm 0.19$ & 0.0053 \\
\hline SA & $58.49^{\mathrm{a}} \pm 0.52$ & $58.28^{a} \pm 0.85$ & $57.94^{\mathrm{ab}} \pm 0.91$ & $58.10^{\mathrm{ab}} \pm 0.60$ & $57.65^{\mathrm{ab}} \pm 0.40$ & $58.64^{a} \pm 0.84$ & $56.08^{b c} \pm 0.59$ & $55.99^{b c} \pm 0.73$ & $55.26^{c} \pm 0.58$ & 0.0057 \\
\hline EV & $41.18^{a} \pm 0.54$ & $40.97^{a} \pm 0.89$ & $40.61^{\mathrm{ab}} \pm 0.94$ & $40.77^{a b} \pm 0.62$ & $40.30^{a b} \pm 0.42$ & $41.34^{a} \pm 0.89$ & $38.67^{b c} \pm 0.61$ & $38.59^{b c} \pm 0.74$ & $37.84^{c} \pm 0.60$ & 0.0060 \\
\hline EW & $45.10^{a} \pm 0.60$ & $44.87^{a} \pm 0.98$ & $44.48^{\mathrm{ab}} \pm 1.03$ & $44.66^{a b} \pm 0.68$ & $44.15^{\mathrm{ab}} \pm 0.45$ & $45.28^{a} \pm 0.97$ & $42.36^{b c} \pm 0.67$ & $42.26^{b c} \pm 0.81$ & $41.44^{c} \pm 0.65$ & 0.0060 \\
\hline HU & $78.62^{\mathrm{ab}} \pm 1.63$ & $79.16^{a} \pm 1.16$ & $78.75^{\mathrm{ab}} \pm 1.34$ & $76.14^{\text {abcd }} \pm 1.24$ & $77.95^{\mathrm{abc}} \pm 0.93$ & $77.60^{\mathrm{abc}} \pm 1.33$ & $74.84^{\mathrm{cd}} \pm 0.63$ & $73.69^{d} \pm 0.85$ & $75.15^{\mathrm{bcd}} \pm 0.45$ & 0.0088 \\
\hline YI & $49.75 \pm 2.81$ & $50.20 \pm 2.29$ & $49.00 \pm 0.53$ & $51.24 \pm 1.16$ & $50.40 \pm 0.77$ & $48.35 \pm 1.73$ & $48.79 \pm 0.37$ & $47.65 \pm 0.24$ & $47.39 \pm 0.33$ & 0.5937 \\
\hline ST & $0.34 \pm 0.01$ & $0.32 \pm 0.00$ & $0.32 \pm 0.01$ & $0.34 \pm 0.01$ & $0.32 \pm 0.01$ & $0.31 \pm 0.01$ & $0.35 \pm 0.01$ & $0.34 \pm 0.01$ & $0.34 \pm 0.02$ & 0.1800 \\
\hline
\end{tabular}

a-d Means in a row with no common superscript differ significantly at $p \leq 0.05$.

Values are least square mean \pm standard error.

RNN = Rhode Island Red $\times$ Naked Neck; BNN = Black Australorp $\times$ Naked Neck; NN = Naked Neck; FR = Free Range; SI = Semi Intensive; I = Intensive; SI = Shape Index; SA = Surface Area $\left(\mathrm{cm}^{2}\right) ; \mathrm{EV}=$ Egg Volume $\left(\mathrm{cm}^{3}\right) ; \mathrm{EW}=$ Egg Weight $(\mathrm{g}) ; \mathrm{HU}=$ Haugh Unit Score; $\mathrm{YI}=$ Yolk Index; ST = Shell Thickness (mm).

Table 6 - Effect of genotype and housing system on egg characteristics at 46 weeks. ${ }^{1}$

\begin{tabular}{|c|c|c|c|c|c|c|c|c|}
\hline \multirow{2}{*}{ Trait } & \multicolumn{3}{|c|}{ Genotype } & \multirow{2}{*}{$p$-value } & \multicolumn{3}{|c|}{ Housing System } & \multirow{2}{*}{$p$-value } \\
\hline & RNN (n=15) & BNN $(n=15)$ & $N N(n=15)$ & & $F R(n=15)$ & $\mathrm{SI}(\mathrm{n}=15)$ & $I(n=15)$ & \\
\hline $\mathrm{SI}$ & $77.10 \pm 0.82$ & $76.60 \pm 1.07$ & $74.21 \pm 1.07$ & 0.1067 & $75.47 \pm 1.40$ & $76.47 \pm 0.77$ & $75.98 \pm 0.84$ & 0.7798 \\
\hline SA & $64.75^{a} \pm 0.84$ & $65.12^{\mathrm{a}} \pm 0.71$ & $59.59^{b} \pm 0.46$ & $<0.0001$ & $63.01 \pm 0.85$ & $63.17 \pm 0.97$ & $63.29 \pm 1.06$ & 0.9619 \\
\hline EV & $47.97^{a} \pm 0.93$ & $48.36^{a} \pm 0.78$ & $42.35^{b} \pm 0.48$ & $<0.0001$ & $46.06 \pm 0.92$ & $46.24 \pm 1.06$ & $46.38 \pm 1.15$ & 0.9566 \\
\hline EW & $55.54^{a}+1.01$ & $52.97^{a} \pm 0.85$ & $46.39^{b} \pm 0.53$ & $<0.0001$ & $50.45 \pm 1.01$ & $50.65 \pm 1.16$ & $50.80 \pm 1.26$ & 0.9563 \\
\hline $\mathrm{HU}$ & $82.44^{a} \pm 0.77$ & $82.12^{a} \pm 0.74$ & $75.38^{b} \pm 0.79$ & $<0.0001$ & $80.45 \pm 1.22$ & $80.12 \pm 0.93$ & $79.37 \pm 1.28$ & 0.6153 \\
\hline YI & $49.20^{\mathrm{a}} \pm 2.72$ & $48.00^{a} \pm 1.90$ & $37.47^{b} \pm 1.51$ & 0.0004 & $43.00 \pm 2.48$ & $43.93 \pm 2.15$ & $47.73 \pm 2.75$ & 0.2384 \\
\hline ST & $0.35^{\mathrm{a}} \pm 0.01$ & $0.32^{b} \pm 0.01$ & $0.28^{c} \pm 0.01$ & $<0.0001$ & $0.32 \pm 0.01$ & $0.33 \pm 0.01$ & $0.31 \pm 0.01$ & 0.2441 \\
\hline
\end{tabular}

acc Means in a row with no common superscript differ significantly at $p \leq 0.05$.

'Values are least square mean \pm standard error.

RNN = Rhode Island Red $\times$ Naked Neck; BNN = Black Australorp $\times$ Naked Neck; NN = Naked Neck; FR = Free Range; SI = Semi Intensive; I =Intensive; SI = Shape Index; SA = Surface Area $\left(\mathrm{cm}^{2}\right) ; \mathrm{EV}=$ Egg Volume $\left(\mathrm{cm}^{3}\right) ; \mathrm{EW}=$ Egg Weight (g); HU = Haugh Unit Score; YI = Yolk Index; ST = Shell Thickness (mm). 
Table 7 - Interaction effects (genotype $\times$ housing system) on egg characteristics at 46 weeks $^{1}$.

\begin{tabular}{|c|c|c|c|c|c|c|c|c|c|c|}
\hline \multirow{2}{*}{ Trait } & \multicolumn{3}{|c|}{ RNN } & \multicolumn{3}{|c|}{ BNN } & \multicolumn{3}{|c|}{$\mathrm{NN}$} & \multirow{2}{*}{$p$-value } \\
\hline & $F R(n=5)$ & $S I(n=5)$ & $I(n=5)$ & $F R(n=5)$ & $\mathrm{SI}(\mathrm{n}=5)$ & $I(n=5)$ & $F R(n=5)$ & $\mathrm{SI}(\mathrm{n}=5)$ & $I(n=5)$ & \\
\hline $\mathrm{SI}$ & $79.09 \pm 1.66$ & $76.17 \pm 1.16$ & $76.03 \pm 1.19$ & $72.25 \pm 2.54$ & $77.36 \pm 0.61$ & $77.20 \pm 2.14$ & $72.05 \pm 2.27$ & $75.87 \pm 2.07$ & $74.72 \pm 0.76$ & 0.2843 \\
\hline SA & $63.79^{\mathrm{a}} \pm 0.95$ & $65.80^{\mathrm{a}} \pm 1.33$ & $64.67^{\mathrm{a}} \pm 2.04$ & $65.91^{\mathrm{a}} \pm 0.70$ & $63.75^{\mathrm{a}} \pm 1.89$ & $65.70^{\mathrm{a}} \pm 0.71$ & $59.32^{b} \pm 0.68$ & $59.96^{b} \pm 0.27$ & $59.49^{b} \pm 1.26$ & 0.0002 \\
\hline EV & $46.89^{a} \pm 1.04$ & $49.12^{\mathrm{a}} \pm 1.50$ & $47.90^{\mathrm{a}} \pm 2.25$ & $49.22^{\mathrm{a}} \pm 0.78$ & $46.88^{a} \pm 2.07$ & $48.99^{a} \pm 0.79$ & $42.07^{b} \pm 0.73$ & $42.73^{b} \pm 0.28$ & $42.26^{b} \pm 1.33$ & 0.0003 \\
\hline EW & $51.36^{a} \pm 1.14$ & $53.80^{a} \pm 1.64$ & $52.47^{a} \pm 2.47$ & $53.91^{\mathrm{a}} \pm 0.85$ & $51.35^{\mathrm{a}} \pm 2.27$ & $53.66^{a} \pm 0.86$ & $46.07^{b} \pm 0.79$ & $46.81^{b} \pm 0.31$ & $46.29^{b} \pm 1.46$ & 0.0003 \\
\hline $\mathrm{HU}$ & $82.49^{a} \pm 1.71$ & $81.75^{\mathrm{a}} \pm 1.54$ & $83.07^{a} \pm 0.84$ & $83.49^{\mathrm{a}} \pm 1.19$ & $81.92^{\mathrm{a}} \pm 1.41$ & $80.95^{a} \pm 1.24$ & $75.36^{b} \pm 1.25$ & $76.70^{b} \pm 0.54$ & $74.09^{b} \pm 1.96$ & $<0.0001$ \\
\hline YI & $50.40^{\mathrm{a}} \pm 5.12$ & $44.00^{\mathrm{abc}} \pm 4.21$ & $53.20^{\mathrm{a}} \pm 4.76$ & $42.40^{\mathrm{abc}} \pm 1.75$ & $48.80^{\mathrm{ab}} \pm 3.77$ & $52.80^{a} \pm 2.62$ & $36.20^{c} \pm 3.18$ & $39.00^{\mathrm{bc}} \pm 2.17$ & $37.20^{\complement} \pm 2.85$ & 0.0044 \\
\hline ST & $0.36^{a} \pm 0.01$ & $0.37^{a} \pm 0.02$ & $0.32^{\mathrm{abc}} \pm 0.02$ & $0.31^{b c} \pm 0.02$ & $0.34^{\mathrm{ab}} \pm 0.02$ & $0.32^{\mathrm{abc}} \pm 0.01$ & $0.28^{c} \pm 0.02$ & $0.29 b^{c} \pm 0.02$ & $0.28^{c} \pm 0.01$ & 0.0012 \\
\hline
\end{tabular}

acc Means in a row with no common superscript differ significantly at $p \leq 0.05$

Values are least square mean \pm standard error.

RNN = Rhode Island Red $\times$ Naked Neck; BNN = Black Australorp $\times$ Naked Neck; NN = Naked Neck; FR = Free Range; SI = Semi Intensive; I = Intensive; SI = Shape Index; SA = Surface Area $\left(\mathrm{cm}^{2}\right) ; \mathrm{EV}=$ Egg Volume $\left(\mathrm{cm}^{3}\right) ; \mathrm{EW}=$ Egg Weight $(\mathrm{g}) ; \mathrm{HU}=$ Haugh Unit Score; YI = Yolk Index; ST = Shell Thickness (mm).

experiment (46 weeks), egg shape index did not differ among housing systems $(p=0.7798)$ or genotypes $(p=0.1067)$ and their interaction was not significant $(p=0.2843)$.

The eggs of RNN and BNN hens had larger egg surface area (26 wks, $p<0.0001 ; 46$ wks, $p<0.0001$ ) than NN hens. The interaction between housing systems and genotypes shows larger egg surface area ( 26 wks, $p=0.0057 ; 46$ wks, $p=0.0002$ ) in RNN hens with in all evaluated systems.

Higher egg volume (26 wks, $p<0.0001 ; 46$ wks, $p<0.0001)$ was determined in RNN and BNN than in NN genotypes. The interaction between factors showed higher egg volume ( 26 wks, $p=0.0060 ; 46$ wks, $p=0.0003$ ) in RNN and BNN hens in all housing systems.

Heavier eggs ( 26 wks, $p<0.0001 ; 46$ wks, $p<0.0001)$ were laid by RNN and BNN hens than NN hens. There was no effect $(p>0.05)$ of housing system on egg weight. However, the interaction between factors showed that the heaviest eggs ( 26 wks, $p=0.0060$; 46 wks, $p=0.0003$ ) were laid by RNN hens with freerange, semi-intensive and intensive systems and BNN in the intensive system.

RNN and BNN eggs had higher Haugh unit score (26 wks, $p=0.0002 ; 46$ wks, $p=<0.0001)$, meaning that their albumen quality was better compared with $N N$ eggs. There was no effect ( $p>0.05)$ of housing system on Haugh units. However, the highest Haugh unit scores ( 26 wks, $p=0.0088 ; 46$ wks, $p<0.0001$ ) were determined RNN and BNN eggs in all housing systems.

Initially, yolk index did not differ among housing systems ( $p=0.3512$ ) or genotypes $(p=0.1883)$, and their interaction was not significant $(p=0.5937)$. However, at the end of the experiment (46 weeks), higher $(p=0.0004)$ yolk index was found in RNN and BNN eggs than in NN eggs. The interaction among factors determined the highest egg yolk index $(p=0.0044)$ in the eggs of RNN and BNN hens reared in the freerange and intensive systems.

At the start of the experiment ( 26 weeks), eggshell thickness was not influenced by housing system $(p=0.0724)$, genotype $(p=0.0787)$ or their interaction $(p=0.1800)$. However, at the end of the experiment (46 weeks), eggs of RNN hens presented thicker eggshells $(p<0.0001)$, followed by those of BNN and RNN hens. The interaction showed that the thickest eggshells $(p=0.0012)$ were produced by RNN and BNN hens reared in the free-range and semi-intensive systems.

\section{Hatching traits}

Hatchability, fertility, and infertile egg rates (\%) were influenced by housing system, genotype, and their interaction, whereas dead-in-shell rate differed among housing systems, but not among genotypes (Tables 8, 9).

Higher hatchability $(p<0.0001)$ was obtained in the free-range system, followed by the semi-intensive and intensive systems. Regarding genotypes, RNN eggs had the highest $(p<0.0001)$ hatchability, followed by BNN and NN. In the interaction between housing system and genotype, eggs of RNN hens in the free-range system presented the highest hatchability $(p<0.0001)$.

Eggs laid by free-range hens had higher $(p<0.0001)$ fertility compared with the semi-intensive and intensive systems. Among genotypes, RNN and BNN had higher $(p<0.0001)$ egg fertility rate than NN. The interaction among factors showed that free-range RNN hens had the highest $(p<0.0001)$ egg fertility rate.

Egg infertility rate was lowest $(p<0.0001)$ in freerange hens, followed by those kept in the semiintensive, and intensive systems. Regarding genotypes, RNN and BNN hens had lower $(p<0.0001)$ egg infertility rate than NN hens. The interaction between factors showed that the lowest fertility rate $(p<0.0001)$ was obtained in eggs from free-range RNN hens. 
Table 8 - Effect of genotype and housing system on hatching traits. ${ }^{1}$

\begin{tabular}{|c|c|c|c|c|c|c|c|c|}
\hline \multirow{2}{*}{ Trait (\%) } & \multicolumn{3}{|c|}{ Genotype } & \multirow{2}{*}{$p$-value } & \multicolumn{3}{|c|}{ Housing System } & \multirow{2}{*}{ P-value } \\
\hline & RNN $(n=15)$ & BNN $(n=15)$ & $N N(n=15)$ & & $\mathrm{FR}(\mathrm{n}=15)$ & $\mathrm{SI}(\mathrm{n}=15)$ & $I(n=15)$ & \\
\hline $\mathrm{HP}$ & $71.57^{a} \pm 1.24$ & $69.24^{b} \pm 1.37$ & $64.14^{c} \pm 1.27$ & $<0.0001$ & $73.61^{a} \pm 0.91$ & $67.28^{b} \pm 1.39$ & $64.07^{c} \pm 0.99$ & $<0.0001$ \\
\hline FP & $87.43^{a} \pm 0.69$ & $86.69^{a} \pm 0.90$ & $81.74^{b} \pm 1.30$ & $<0.0001$ & $88.42^{\mathrm{a}} \pm 0.80$ & $84.71^{b} \pm 0.83$ & $81.72^{b} \pm 1.35$ & $<0.0001$ \\
\hline IP & $12.57^{b} \pm 0.69$ & $13.31^{b} \pm 0.90$ & $18.26^{a} \pm 1.30$ & $<0.0001$ & $11.58^{b} \pm 0.80$ & $15.29^{a} \pm 0.83$ & $17.28^{a} \pm 1.35$ & $<0.0001$ \\
\hline DG & $7.91 \pm 0.45$ & $8.45 \pm 0.76$ & $8.66 \pm 0.64$ & 0.6430 & $7.50 \pm 0.50$ & $8.28 \pm 0.67$ & $9.24 \pm 0.63$ & 0.1168 \\
\hline DIS & $7.94 \pm 0.49$ & $9.00 \pm 0.84$ & $8.94 \pm 0.55$ & 0.4154 & $7.31^{b} \pm 0.51$ & $9.16^{a} \pm 0.72$ & $9.41^{\mathrm{a}} \pm 0.57$ & 0.0456 \\
\hline
\end{tabular}

a-c Means in a row with no common superscript differ significantly at $p \leq 0.05$.

VValues are least square mean \pm standard error.

RNN = Rhode Island Red $\times$ Naked Neck; BNN = Black Australorp $\times$ Naked Neck; NN = Naked Neck; FR = Free Range; SI = Semi Intensive; I = Intensive; Hp=Hatchability Percent; Fp=Fertility Percent; I $\mathrm{p}=$ Infertile Percent; DG = Dead Germ Percent; DIS = Dead in Shell Percent.

Table 9 - Interaction effects (genotype $\times$ housing system) on hatching traits ${ }^{1}$.

\begin{tabular}{|c|c|c|c|c|c|c|c|c|c|c|}
\hline \multirow{2}{*}{ Trait (\%) } & \multicolumn{3}{|c|}{ RNN } & \multicolumn{3}{|c|}{ BNN } & \multicolumn{3}{|c|}{$\mathrm{NN}$} & \multirow{2}{*}{$p$-value } \\
\hline & $F R(n=5)$ & $\mathrm{SI}(\mathrm{n}=5)$ & $I(n=5)$ & $F R(n=5)$ & $\mathrm{SI}(\mathrm{n}=5)$ & $I(n=5)$ & $\mathrm{FR}(\mathrm{n}=5)$ & $\mathrm{SI}(\mathrm{n}=5)$ & $I(n=5)$ & \\
\hline $\mathrm{HP}$ & $77.29^{a} \pm 1.16$ & $69.08^{c} \pm 1.22$ & $68.35^{c} \pm 1.03$ & $73.43^{b} \pm 0.44$ & $70.60^{b c} \pm 2.55$ & $63.69^{d} \pm 0.75$ & $70.11 b c \pm 0.85$ & $62.16^{d} \pm 1.47$ & $60.16^{d} \pm 0.43$ & $<0.0001$ \\
\hline FP & $90.23^{a} \pm 0.84$ & $86.46^{\mathrm{ab}} \pm 0.79$ & $85.60^{b} \pm 0.81$ & $88.35^{\mathrm{ab}} \pm 1.93$ & $86.37^{\mathrm{ab}} \pm 0.44$ & $85.34^{b} \pm 1.87$ & $86.69^{\mathrm{ab}} \pm 0.89$ & $81.31^{c} \pm 1.47$ & $77.23^{d} \pm 1.89$ & $<0.0001$ \\
\hline IP & $9.77 d \pm 0.84$ & $13.54^{\mathrm{cd}} \pm 0.79$ & $14.40^{c} \pm 0.81$ & $11.65^{\mathrm{cd}} \pm 1.93$ & $13.63^{\mathrm{cd}} \pm 0.44$ & $14.66^{c} \pm 1.87$ & $13.31^{\mathrm{cd}} \pm 0.89$ & $18.69^{b} \pm 1.47$ & $22.77^{\mathrm{a}} \pm 1.89$ & $<0.0001$ \\
\hline DG & $6.62 \pm 0.98$ & $8.71 \pm 0.32$ & $8.41 \pm 0.63$ & $7.24 \pm 0.95$ & $6.98 \pm 1.12$ & $11.12 \pm 1.09$ & $8.65 \pm 0.50$ & $9.14 \pm 1.64$ & $8.19 \pm 1.11$ & 0.1017 \\
\hline DIS & $6.32 \pm 0.73$ & $8.67 \pm 0.86$ & $8.84 \pm 0.46$ & $7.68 \pm 1.10$ & $8.78 \pm 1.90$ & $10.53 \pm 1.28$ & $7.94 \pm 0.81$ & $10.01 \pm 0.88$ & $8.87 \pm 1.08$ & 0.2643 \\
\hline
\end{tabular}

a-d Means in a row with no common superscript differ significantly at $p \leq 0.05$.

'Values are least square mean \pm standard error.

RNN = Rhode Island Red $\times$ Naked Neck; BNN = Black Australorp $\times$ Naked Neck; NN = Naked Neck; FR = Free Range; SI = Semi Intensive; I = Intensive; Hp = Hatchability Percent; $F p=$ Fertility Percent; Ip = Infertile Percent; DG = Dead Germ Percent; DIS = Dead in Shell Percent.

Lower dead-in-shell rate was obtained in free-range hens ( $p=0.0456)$ compared with those maintained in the semi-intensive and intensive systems. Dead-in-shell rate was not influenced by genotype $(p=0.4154)$ and its interaction with housing system was not significant $(p=0.2643)$.

No significant differences in embryo mortality were found among housing systems $(p=0.1168)$ or genotypes $(p=0.6430)$ and their interaction was not significant $(p=0.1017)$.

\section{DISCUSSION}

The present study evaluated the productive performance, egg characteristics and hatching traits of three chicken genotypes under different housing systems. Hens maintained in the intensive system were heavier in weeks 26 and 46 and showed better productive performance (higher egg weight, egg mass, and egg production, \%) than those reared in the semi-intensive and free-range systems. The most likely explanation for the lower body weight of freerange hens is their higher activity and movement in free-range area, which ultimately burns more calories. The observed differences in the productive potential are consistent with the findings of Rehman et al. (2016), who found better productive performance of Indigenous Aseel chicken reared under confined and semi-intensive systems. Similarly, Hameed et al. (2012) reported better productive performance of different broiler breeder strains maintained under a controlled housing system.

BNN hens were heavier both in weeks 26 and 46 and showed better productive performance compared with RNN and NN chickens. The higher productive potential of BNN hens may be attributed to the combination of Black Australorp and Naked Neck genes, as the Black Australorp is popular for its egg laying potential and Naked Neck for its adaptability to extreme weather conditions, resulting in exceptional egg production and excellent adaptability to local climatic conditions of its cross. These findings arein agreement with the study of Rehman et al., (2016), who found variation in productive performance among different varieties of Aseel chickens (Lakha, Mushki, Peshawari and Sindhi) and reported higher egg production in Peshawari and Sindhi varieties.

Although higher egg shape index was obtained in RNN and BNN hens compared with NN hens at the start of the experiment (26 weeks), no differences among housing systems and genotypes were detected at the end of the experiment (46 weeks). The lack of influence of housing system on egg shape index is in agreement with Rehman et al. (2017), who did not find any egg shape index differences among Aseel hens reared in confinement, semi-intensive, or and free- 
range production systems. However, variation exists among different varieties of Native Aseel chickens in Pakistan. Literature studies have also reported egg shape index variations among indigenous chickens and laying hens (Van Den Brand et al., 2004; Rayan et al., 2010). In the present study, the eggs of RNN and BNN hens har larger surface area and volume compared with those of NN hens. This difference is consistent with the findings of Rehman et al. (2017) and Rayan et al. (2010), who found variations in egg surface area and volume among different varieties of Aseel chicken and broiler breeder strains, respectively. It was further noted in that brown broiler breeder eggs had larger egg surface area and volume compared with white egg layers (Rayan et al., 2010). Similar studies reported variation in egg surface area among different strains (Anderson et al., 2004) and breeds (Islam et al., 2010) of chickens.

Higher Haugh unit scores were obtained in eggs of RNN and BNN hens, which indicates that their albumen quality was better compared with eggs of NN hens. This result is in line with the findings of Dunga (2013), who found variation in Haugh unit scores between Naked Neck and Aseel chickens. However, Rajkumar et al. (2009) did not report any-significant Haugh unit differences among different chicken genotypes.

Initially, yolk index and eggshell thickness did not differ among housing systems, genotypes, or their interaction. However, at the end of the experiment (46 weeks), higher yolk index and eggshell thickness were determined in the eggs of RNN and BNN hens compared with those of NN hens. Rajkumar et al. (2009) also found lower egg yolk index in Naked Neck chickens than normal feathered chickens. Similarly, Dunga (2013) reported variation in yolk index between naked neck and frizzled chickens in India. However, numerous studies did not find any egg shell thickness differences among different chicken genotypes (Hocking et al., 2003; Dukic-Stojcic et al., 2009; Rehman et al., 2017).

Higher fertility and hatchability rates were obtained free-range hens followed by those housed in the semiintensive and intensive systems. This in agreement with the findings of Oke et al. (2015), who reported that rearing guinea fowl on free range improved their reproductive and physiological performance. On the other hand, Mothibedi et al. (2016) reported better semen quality traits and ultimately better fertility in Botswana chicken genotypes reared in an intensive housing system.

Regarding genotypes, RNN hens had the highest fertility and hatchability rates. Furthermore, RNN and BNN hens laid less infertile eggs than NN hens.
This result agrees with the findings of Adeleke et al. (2012), who reported variation in hatching traits between frizzled and normal feathered chickens, where frizzled and normal feather chickens presented 90.5 and $84.8 \%$ fertility, respectively, and Anak Titan and Naked Neck showed 80.1 and $76.7 \%$ hatchability, respectively. Those authors found that naked-neck chickens had highest dead-in-shells, while Anak Titan had highest embryo mortality.

\section{CONCLUSIONS}

Free-range and semi-intensive systems positively influence productive performance, egg quality and hatching traits. Regarding genotypes, RNN and BNN crossbreds perform better than NN purebreds. Hence, RNN and BNN chickens can be useful for rural poultry farmers and can be reared under semi-intensive or free-range housing systems.

\section{ACKNOWLEDGEMENTS}

This study was financially supported by Pakistan Agricultural Research Council, ALP fund (Project No. AS-135).

\section{REFERENCES}

Adeleke MA, Peters SO, Ozoje MO, Ikeobi CON, Bamgbose AM, Adebambo OA. Effect of crossbreeding on fertility, hatchability and embryonic mortality of Nigerian local chickens. Tropical Animal Health and Production 2012;44:505-510.

Ahmad S, Mahmud A, Hussain J, Javed K. Morphological and carcass traits of three chicken genotypes under free-range, semiintensive, and intensive housing systems. Turkish Journal of Veterinary and Animal Sciences 2019a;43. 342-352.

Ahmad S, Mahmud A, Hussain J, Javed K. Morphometric traits, serum chemistry and antibody response of three chicken genotypes under free-range, semi-intensive and intensive housing systems. Brazilian Journal of Poultry Science 2019b;21(1):1-8.

Anderson KE, Tharrington JB, Curtis PA, Jones FT. Shell characteristics of eggs from historic strains of single comb White Leghorn chickens and the relationship of egg shape to shell strength. International Journal of Poultry Science 2004;3:17-19.

Bain MM. Recent advances in the assessment of egg shell quality and their future application. World's Poultry Science Journal 2005;61:268-277.

Chen X, Jiang W, Tan HZ, Xu GF, Zhang XB, Wei S, et al. Effects of outdoor access on growth performance, carcass composition, and meat characteristics of broiler chickens. Poultry Science 2013;92:435-443.

Dukic-Stojcic M, Peric L, Bjedov S, Milosevic N. The quality of table eggs produced in different housing systems. Biotechnology Animal Husbandry 2009;25:1103-1108.

Dunga GT. The effect of the naked neck ( $\mathrm{Na}$ ) and frizzling genes on the fertility, hatchability, egg quality and pterylosis of locally developed commercial layer parent lines [PhD thesis]. Kumasi (RSA): Kwame Nkrumah University Science Technology; 2013. 
Economic Survey of Pakistan. Agriculture. III. Livestock and Poultry. Pakistan Economic Survey; 2017-18. Available from: http://www.finance.gov. pk/survey/chapters_18/02-Agriculture.pdf.

Gikunju MM, Kabuage LW, Wachira AM, Oliech GW, Gicheha MG. Evaluation of pure breeds, crossbreeds and indigenous chicken egg quality traits in Kenya. Livestock Research for Rural Development 2018;30:170.

Hameed T, Bajwa MA, Abbas F, Sahota AW, Tariq MM, Khan SH, et al. Effect of housing system on production performances of different broiler breeder strains. Pakistan Journal of Zoology 2012;44(6):1683-1687.

Hocking P, Bain M, Channing C, Fleming R, Wilson S. Genetic variation for egg production, egg quality and bone strength in selected and traditional breeds of laying fowl. British Poultry Science 2003;44:365373.

Iqbal A, Akram M, Sahota AW, Javed K, Hussain J, Sarfraz Z, et al. Laying characteristics and egg geometry of four varieties of indigenous Aseel chicken in Pakistan. Journal of Animal and Plant Sciences 2012;22(4):848-852

Islam SM, Dutta RK. Egg quality traits of indigenous, exotic and cross bred chickens (Gallus domesticus L.) in Rajshahi, Bangladesh. Journal of Life Earth Science 2010;5:63-67.

Lay Jr. DC, Fulton RM, Hester PY, Karcher DM, Kjaer JB, Mench JA, et al Hen welfare in different housing systems. Poultry Science 2011;90(1):278294.

Leeson S, Summers JD. Commercial poultry nutrition. $3^{\text {rd }}$ ed. Nottingham: Nottingham University Press; 2005. p. 297-305.

Leinonen I, Williams AG, Kyriazakis I. The effects of welfare-enhancing system changes on the environmental impacts of broiler and egg production. Poultry Science 2014;93(2):256-266.

Lemlem A, Tesfay Y. Performance of exotic and indigenous poultry breeds managed by smallholder farmers in northern Ethiopia. Livestock Research and Rural Development 2010;22(7).

Mothibedi K, Nsoso SJ, Waugh EE, Kgwatalala PM. Semen characteristics of purebred naked neck tswana and black australorp $\times$ naked neck tswana crossbred chickens under an intensive management system in botswana. American Journal of Research and Communication 2016;4(10):38-47.

NRC - National Research Council. Nutrient requirement table of poultry. $9^{\text {th }}$ ed. Washington: National Academy Press; 1994.
Oke OE, Adejuyigbe AE, Idowu OP, Sogunle OM, Ladokun AO, Oso AO, et al. Effects of housing systems on reproductive and physiological response of guinea fowl (Numida Meleagris). Journal of Applied Animal Science 2015;8(1):47-55.

Peters SO, Shoyebo OD, Ilori BM, Ozoje MO, Ikeobi CON, Adebambo OA. Semen quality traits of seven strains of chickens raised in the humid tropics. International Journal of Poultry Science 2008;7(10):949-953.

Rajkumar U, Sharma RP, Rajaravindra KS, Niranjan M, Reddy BLN, Bhattacharya TK, et al. Effect of genotype and age on egg quality traits in Naked Neck chicken under tropical climate from India. International Journal of Poultry Science 2009;8:1151-1155.

Rayan GN, Galal A, Fathi MM, ElAttar AH. Impact of layer breeder flock age and strain on mechanical and ultra-structural properties of eggshell in chicken. International Journal of Poultry Science 2010;9:139-147.

Rehman MS, Mahmud A, Mehmood S, Pasha TN, Hussain J, Khan MT. Comparative evaluation of egg morphometry and quality in Aseel hens under different rearing systems. Journal of Applied Poultry Research 2017;26:401-409.

Rehman MS, Mahmud A, Mehmood S, Pasha TN, Javed K, Hussain J, et al. Production performance of aseel chicken under free-range, semiintensive and confinement rearing systems. Journal of Animal and Plant Sciences 2016;26(6):1589-1596.

Sadef S, Khan MS, Rehman MS. Indigenous chicken production in Punjab: a detailed survey through participatory rural appraisals. Journal of Animal and Plant Sciences 2015;25:1273-1282.

Sekeroglu A, Altuntas E. Effects of egg weight on egg quality characteristics. Journal of Science Food and Agriculture 2009;89:379-383.

Shafik BMN, El-Bayomi KM, Sosa GA, Osman AMR. Effect of crossing Fayoumi and Rhode Island Red on growth performance, egg and reproductive traits under Egyptian conditions. Benha Veterinary Medical Journal 2013;24(2):11-18

Tukey JW. The problem of multiple comparisons. In: The collected works of John W. Tukey VIII. Multiple comparisons. New York: Chapman and Hall; 1953.

Van Den Brand H, Parmentier HK, Kemp B. Effect of housing system (outdoor vs cages) and age of laying hens on egg characteristics. British Poultry Science 2004;45:745-752. 
